\title{
Management of giant hepatic hemangiomas: a case report
}

Keywords: giant hepatic hemangioma, asymptomatic, surgical treatment versus observation

\section{Introduction}

Haemangioma are by far the most common benign tumour affecting the liver, they are especially prevalent in women. ${ }^{1}$ In most cases, hemangiomas are small and asymptomatic. The widespread use of noninvasive abdominal imaging modalities has led to an increased detection of various lesions. The relation between the complaints of the patient and the lesion detected however is often unclear. Nevertheless, clinical symptomatology and effects are mostly due to size of these benign lesions. Arbitrary giant haemangiomas have been defined as larger than 5 centimeter $(\mathrm{cm})$ in diameter. Larger and multiple lesions especially in the left liver lobe might cause (acute) symptoms due to compression of adjacent structures, thrombosis or infarction of the lesion. Hemorrhage has been described as well. ${ }^{2}$ Rarely, large hemangiomas rupture, either spontaneously or following blunt abdominal trauma. Surgical treatment then is inevitable and may lead to complete relief of symptoms. The management of large hepatic hemangiomas in asymptomatic patients, however, is more controversial, as discussed by Duxbury et al. ${ }^{3}$ and Schnelldorfer et al. ${ }^{4}$

\section{Case report}

A 55-year old woman presented at the outpatients clinic with right upper quadrant pain and abdominal distention. A year before she was diagnosed with endometrial carcinoma, stage IA, and underwent abdominal hysterectomy and removal of both ovaries. She had not received oral contraceptives in the past. Pre-operative evaluation for extent of disease had shown several lesions in the liver suspicious of hemangiomas on ultrasonography. The two largest lesions were 19 and $5 \mathrm{~cm}$ respectively. Computed tomography and magnetic resonance imaging supported the diagnosis. The giant hemangioma was located in an enlarged right lobe of the liver and the smaller one in the left lobe (Figure 1).

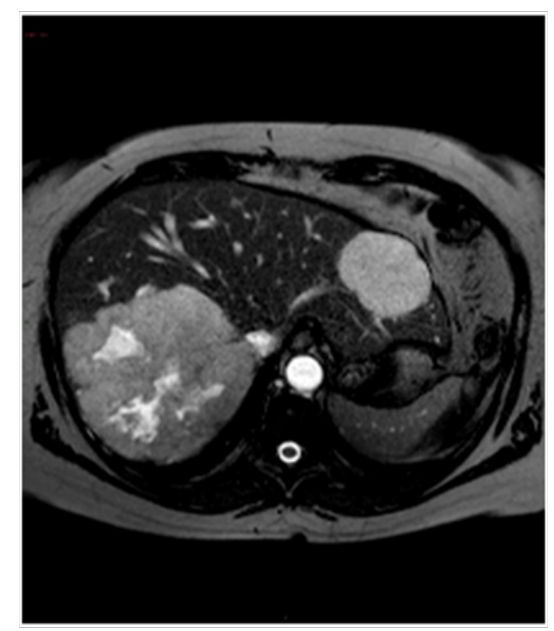

Figure I MRI with two haemangiomas in the right and left liver lobe.

\author{
Volume 2 Issue 4 - 2015 \\ Chris M Kievit, Rutger Jan Nuis, Adriaan \\ Dees \\ Department of Internal Medicine, Ikazia Hospital, Netherlands
}

\begin{abstract}
Correspondence: Adriaan Dees, Department of Internal Medicine, Ikazia Hospital, Montessoriweg I, 3083 AN, Rotterdam, Netherlands, Tel +3 I (0) I02975 I36, Fax+3 I (0) 104859959, Email adees@planet.nl
\end{abstract}

Received: April 28, 2015 | Published: July 16, 2015

The patient told that she suffered from abdominal pain for almost two years. She described it as a dull, annoying pain in the right upper quadrant. The pain was related to exercise. Her defecation pattern had been normal and remained unchanged over the years. She didn't report episodes of fever. At physical examination there were no signs of lymphadenopathy. The edge of the right lobe of the liver reached $4 \mathrm{~cm}$ below the costal margin. Examination of the skin didn't demonstrate bleeding sites. The patient's body mass index was 30 . Laboratory investigation showed normal levels of fibrinogen and platelets. Liver function tests were all normal, serum alkaline phosphatase 41U/1 (n 25-120) and Gamma GT 30U/1 $(\mathrm{n}<35)$, which remained unchanged over time. There were no signs of coagulopathy. Without specific treatment or intervention, the patient had no complications over a follow-up period of four and a halve year.

\section{Discussion}

Hepatic hemangiomas are the most common focal liver lesions, often detected incidentally, with a reported incidence rate of approximately $2 \%$, Choi et al. ${ }^{5}$ and Gandolfi et al. ${ }^{6}$ At presentation there were no signs of severe symptoms or complications, which can occur in the setting of Kasabach-Merritt syndrome, a condition associated with a consumptive coagulopathy, as reported by Malagari et al. ${ }^{7}$ A large retrospective cohort study by Schnelldorfer et al., ${ }^{4}$ reported potentially life-threatening complications in $2 \%$ of 233 nonoperative patients with giant hepatic hemangioma during a follow-up of 11years. In the small number of patient who underwent surgery for potentially life-threatening complications, in case of hepatic hemorrhage or rupture, perioperative mortality was $0 \%$. Size of hemangiomas in this study was not associated with adverse events, confirming data from an earlier study by Terkivatan et al. ${ }^{8}$ Giant hemangiomas have been reported up to $27 \mathrm{~cm}$ in diameter.

Besides conservative management hepatic hemangiomas have been treated with a wide array of therapies. Traditionally, surgical resection and surgical enucleation are the treatments of choice, Singh et al. ${ }^{9}$ However, it might be very difficult, even for experienced clinicians, to decide whether a patient should be operated or not. A well designed prospective trial hasn't been performed yet. On the 
other hand perioperative complications have been reported. In the same recent large cohort study by Schnelldorfer et al., ${ }^{4}$ 56patients underwent surgery primarily. In $14 \%$ of these patients perioperative complications have been reported, including potentially lifethreatening complications in $7 \%$. These findings all resemble previous data in literature, as reported by Pietrabissa et al., ${ }^{10}$ Yoon et al. ${ }^{11}$ and Herman et al. ${ }^{12}$ None of the operative patients had persistent or new onset of hemangioma associated symptoms after surgical resection. Minimally invasive therapies for hepatic hemangioma include arterial embolization and radiofrequency ablation, Malagari et al. ${ }^{7}$ and Gao et al. ${ }^{13}$ Orthotopic liver transplantation has been performed as treatment in rare circumstances, Vagefi et al. ${ }^{2}$

\section{Conclusion}

The management of large hemangiomas should be conservative, especially in asymptomatic lesions. Indications for surgical or angiographic intervention include: bleeding, Diffuse Intravascular Coagulation (DIC), progressive abdominal pain and high-output cardiac failure. Taking into account the absence of severe symptoms and patients history, we closely observed the patient for more than five years. Until now, the hemangiomas haven't changed in size and no complications or severe symptoms have occurred.

\section{Acknowledgements}

None

\section{Conflict of interest}

The author declares no conflict of interest.

\section{References}

1. Caseiro AF, Brito J, Araujo AE, et al. Liver haemangioma: common and uncommon findings and how to improve the differential diagnosis. Eur Radiol. 2007;17(6):1544-1554.
2. Vagefi PA, Klein I, Gelb B, et al. Emergent orthotopic liver transplantation for hemorrhage from a giant cavernous hepatic hemangioma:case report and review. J Gastrointest Surg. 2011;15(1):209-214.

3. Duxbury MS, Garden OJ. Giant haemangioma of the liver: observation or resection? Digestive Surgery. 2010;27(1):7-11.

4. Schnelldorfer T, Ware AL, Smoot R, et al. Management of giant hemangioma of the liver: resection versus observation. J Am Coll Surg. 2010;211(6):724-730.

5. Choi BY, Nguyen MH. The diagnosis and management of benign hepatic tumors. J Clin Gastroenterol. 2005;39(5):401-412.

6. Gandolfi L, Leo P, Solmi L, et al. Natural history of hepatic haemangiomas: clinical and ultrasound study. Gut. 1991;32(6):677-680.

7. Malagari K, Alexopoulou E, Dourakis S, et al. Transarterial embolization of giant liver hemangiomas associated with Kasabach-Merrit syndrome; a case report. Acta Radiol. 2007;48(6):608-612.

8. Terkivatan T, Vrijland WW, Den Hoed PT, et al. Size of lesion is not a criterion for resection during management of giant liver haemangioma. Br J Surg. 2002;89(10):1240-1244.

9. Singh RK, Kapoor S, Sahni P, et al. Giant haemangioma of the liver: is enucleation better than resection? Ann $R$ Coll Surg Engl. 2007;89(5):490-493.

10. Pietrabissa A, Giulianotti P, Campatelli A, et al. Management and followup of 78 giant haemangiomas of the liver. Br J Surg. 1996;83(7):915918

11. Yoon SS, Charny CK, Fong Y, et al. Diagnosis, management, and outcomes of 115 patients with hepatic hemangioma. J Am Coll Surg. 2003;197(3):392-402.

12. Herman P, Costa ML, Machado MA, et al. Management of hepatic hemangiomas: a 14-year experience. J Gastrointest Surg. 2005;9(6):853-859.

13. Gao J, Ke S, Ding XM, et al. Radiofrequency ablation for large hepatic hemangiomas: initial experience and lessons. Surgery. 2013;153(5):7885 . 